\title{
Das Familienbild des ABGB und die Lebenssituation von Scheidungs- und Nachscheidungsfamilien
}

This article addresses the question as to how best to characterize the relationship between the image of families promoted in the Austrian General Civil Code $(A B G B)$ and the empirically assessable living conditions of families in Austria. The focus is on the instability of families and post-divorce families' living situations. The relationship between the normative requirements of family law and the empirical findings of family research is shown to be changeful. Normative demands in part adhere to empirically and theoretically obsolete concepts (e.g. marriage and household centeredness). In turn, legal norms generated in other areas prove to be ahead of families' everyday realities (e.g. cooperative role divisions). Examples are drawn upon from the following areas: The arrangement of nonmarital parenthood following separation, the settlement of post-divorce parenthood, and arrangements applying to the living realities of single-parent families and stepfamilies. Law will be increasingly called to attune to instability and discontinuous dynamics.

Das österreichische Ehe- und Familienrecht, wie es im Jahre 1811 im ABGB normativ festgeschrieben wurde, ist in Teilen bis heute in der ursprünglichen Fassung erhalten. Aus soziologischer Sicht wirft die Beständigkeit dieser Rechtsgrundlage die Frage auf, inwiefern soziale Institutionen, die einem starken Wandel unterworfen sind, damit angemessen erfasst werden können. Die Familie stellt eine solche soziale Institution dar und hat in den vergangenen 200 Jahren, besonders seit der Mitte des 20. Jahrhunderts, tiefgreifende Veränderungen erfahren. Der vorliegende Beitrag geht der Frage nach, wie das Verhältnis zwischen dem Familienbild des ABGB und den empirisch darstellbaren Lebensverhältnissen von Familien in Österreich charakterisiert werden kann. Die (In-)Stabilität von Familien und die Lebenssituation von Nachscheidungsfamilien stehen dabei im Mittelpunkt. Zur Beantwortung der Fragestellung werden familiensoziologische Befunde und sta- tistische Daten verwendet, weiters wurden Leitfaden-Interviews mit 14 JuristInnen ${ }^{1}$ geführt und inhaltsanalytisch ausgewertet (MAYRING 2003).

\section{Die bürgerliche Kernfamilie als Leitbild}

$\S 44$ ABGB legt fest: „Die Familien-Verhältnisse werden durch den Ehevertrag gegründet. In dem Ehevertrage erklären zwei Personen verschiedenen Geschlechtes gesetzmäßig ihren Willen, in unzertrennlicher Gemeinschaft zu leben, Kinder zu zeugen, sie zu erziehen, und sich gegenseitig Beistand zu leisten." Diese programmatische Definition von Familie fokussiert auf Ehe und Generationendifferenzierung und

\footnotetext{
${ }^{1}$ Die Interviews fanden zwischen März und Juni 2011 statt. Mein herzlicher Dank gilt allen Interviewpartnern und Interviewpartnerinnen.
} 
geht damit vom Leitbild der bürgerlichen Kernfamilie aus, die durch folgende Merkmale gekennzeichnet ist (HILL, KOPP 2006; HUININK, KONIETZKA 2007): Die Familie basiert auf dem exklusiven, durch Eheschließung legitimierten Zusammenleben von zwei (gegengeschlechtlichen) Elternteilen mit ihren leiblichen Kindern. Sie zielt auf Dauer und ist nicht beliebig kündbar. Während der Mann die Ernährerrolle innehat und für die materielle Versorgung der Familie sowie den Außenbereich zuständig ist, füllt die Frau die Hausfrauenrolle aus und übernimmt im Innenbereich die Versorgung von Kindern und Haushalt („bread-winner" und ",home-maker"). Aus dieser Rollen- und Aufgabenteilung resultiert eine klare Machtverteilung: Die Entscheidungsmacht liegt beim Mann, sein Autoritätsanspruch gegenüber Frau und Kindern wird als natürlich betrachtet.

Bereits zur Entstehungszeit des ABGB konnte dieses Familienbild nur von einem kleinen Teil der Bevölkerung gelebt werden. Heiratsverbote, erbrechtliche Regelungen und ökonomische Faktoren führten dazu, dass ein großer Teil der Bevölkerung unverheiratet blieb und das Heiratsalter relativ hoch war. Erscheinungsformen von Familien waren stark differenziert und von großen regionalen und schichtspezifischen Unterschieden geprägt (GESTRICH 2008; MitTERAUER 2009).

Erst in der Mitte des 20. Jahrhunderts war es einem größeren Teil der Bevölkerung erstmals möglich, das Konzept der bürgerlichen Kernfamilie in der eigenen Biographie umzusetzen: Eheschließungs- und Geburtenraten waren hoch, Heiratsalter und Scheidungsraten niedrig, das männliche Ernährermodell war normativ anerkannt und wurde von einem großen Teil der Familien gelebt. Das Leitbild, welches Eingang in die Konstruktion des ABGB gefunden hatte, ließ sich nun auch in der empirischen Realität abbilden. Diese Zeitspanne wird als "Goldenes Zeitalter der Familie" bezeichnet und war historisch ausgesprochen kurz, nämlich von den 1950er bis zu den 1970er Jahren (SIEDER 1985).

Dennoch hat das Konzept der Kernfamilie nach wie vor weitreichende Bedeutung, da es vielfach die normative Folie darstellt, vor der Familienleben betrachtet wird (ZARTLER 2012) - auch wenn Lebensverläufe heute eher durch Vielfalt und Dynamik gekennzeichnet sind und es, wie bereits zur Entstehungszeit des ABGB, eine Vielfalt von Familienformen gibt. Während BECKGERNSHEIM $(2009,23)$ diesbezüglich von einer "neuen Unordnung" spricht, bezeichnen SCHNeIDeR, MAtTHIAS-BleCK $(1999,191)$ diesen Prozess als „Rückkehr zur Normalität der Vielfalt", da die Situation der 1950er Jahre mit der starken Monopolstellung eines Lebensentwurfs auch im historischen Rückblick eine untypische Situation darstellt (MITTERAUER 2009).

\section{Dynamik und Instabilität im Familienleben}

Die programmatische Definition von Familie, wie sie im $\S 44$ ABGB festgelegt ist, kann der Realität aktuellen Familienlebens kaum gerecht werden. Familie umfasst heute nicht zwingend "zwei Personen verschiedenen Geschlechts". Heteronormative Konzepte werden zunehmend hinterfragt (Oswald, KUVALANKA 2008), und seit 1. Jänner 2010 haben in Österreich homosexuelle Paare die Möglichkeit, ihre Partnerschaft eintragen $\mathrm{zu}$ lassen. ${ }^{2}$ Partnerschaft ist weiters nicht auf die Zeugung von Kindern ausgerichtet, manche Paare bleiben aus unterschiedlichen Gründen kinderlos (BEHAM 2010). Außerdem sind die in einer Familie lebenden Kinder nicht unbedingt gemeinsame Kinder des Paares (s.u.). Ein Ehevertrag ist immer seltener die Basis der Familienbeziehungen: während die Institutionalisierung von Partnerbeziehungen durch eine

\footnotetext{
2 EPG 2009, BGBl. I 2009/135. Im Jahr 2010 haben 705 Paare von dieser Möglichkeit Gebrauch gemacht, im Jahr 2011 dann 433 Paare.
} 
Eheschließung an quantitativer Bedeutung und normativer Verbindlichkeit verloren hat, wurden die rechtlich kaum formalisierten NEL zu einer Standardpassage in der Beziehungsbiographie $^{3}$. Sie haben sich quantitativ stark verbreitet $^{4}$ und als normativ akzeptierte Lebensform, auch für das Leben mit Kindern, etabliert: vier von zehn Kindern werden unehelich geboren, erste Kinder sogar mehrheitlich ${ }^{5}$ (MÜNZ, REITERER 2010, 46). Obwohl seltener und später geheiratet wird, bleibt aber die Heiratsneigung weiterhin hoch, und nach einer Scheidung werden häufig Zweit- oder Dritt-Ehen geschlossen: in gut einem Drittel $(34,4 \%)$ aller neu geschlossenen Ehen war zumindest einer der beiden Partner davor verheiratet. ${ }^{6}$

Schließlich stehen dem „Willen, in unzertrennlicher Gemeinschaft zu leben" steigende Scheidungsraten gegenüber. Nach dem historischen Tiefststand im Jahr 1957 (13,7 \%) betrug die Gesamtscheidungsrate im Jahr 1970 18,1\% und stieg bis zum Höchststand im Jahr 2007 (49,5\%) an. In den letzten Jahren sank die Gesamtscheidungsrate und liegt aktuell (2011) bei $43 \%^{7}$

\footnotetext{
${ }^{3}$ Am häufigsten sind NEL zwischen 25 und 29: Jeder fünfte Mann (21\%) und jede vierte Frau (26\%) dieser Altersgruppe lebt unverheiratet mit einem/einer PartnerIn zusammen. 1981 galt dies für $5 \%$ der Männer und $4 \%$ der Frauen (Statistik Austria 2012c, 23).

${ }^{4}$ NEL ohne Kinder sind zwischen 1985 und 2011 von 2,2 \% auf 8,2\% aller Familien angestiegen; NEL mit Kindern von 1,3\% auf 6,3\% (Statistik Austria 2012c, 23).

${ }^{5}$ Allerdings sind auch die Legitimierungsquoten hoch: etwa jedes zweite unehelich geborene Kind wird durch eine Eheschließung der Eltern legitimiert (§ 161b ABGB) (MÜNZ, REITERER 2010, 46).

${ }^{6} \mathrm{Im}$ Jahr 1970 traf dies auf weniger als ein Viertel (21\%) zu (siehe

http://www.statistik.at/web_de/statistiken/bevoelkeru ng/eheschliessungen/023945.html sowie

http://www.statistik.at/web_de/statistiken/bevoelkeru ng/demographische_masszahlen/demographische_in dikatoren/023577.html (jeweils 25. 8. 2011)

${ }^{7}$ Die Gesamtscheidungsrate gibt die Wahrscheinlichkeit an, mit der im jeweiligen Jahr geschlossene Ehen
}

(Statistik Austria 2012a, 91; Statistik Austria 2012b). Eine Betrachtung auf Personenebene zeigt, dass $11 \%$ der 30- bis 69-jährigen ÖsterreicherInnen geschieden sind und ein Viertel dieser Geschiedenen unverheiratet mit einem/einer neuen PartnerIn zusammenlebt. ${ }^{8}$ Daten zur Anzahl von Trennungen nichtehelicher Lebensgemeinschaften sind für Österreich nicht verfügbar. Internationale Befunde zeigen, dass diese eine höhere Trennungsrate haben als Ehen, was mit niedrigeren Trennungskosten aufgrund der geringeren spezifischen Investitionen erklärt werden kann (BOUCHARD 2006). Jedes vierte geschiedene Paar des Jahres 2011 war kinderlos. Dennoch erlebten 13.300 minderjährige Kinder die Scheidung ihrer Eltern. Das „Scheidungsrisiko" aus Sicht der Kinder, d.h. die Wahrscheinlichkeit, vor dem 18. Geburtstag eine elterliche Scheidung zu erleben, betrug $20 \%$. Die Ergebnisse zur Kontakthäufigkeit zwischen Kind und nicht-residenziellem Elternteil zeigen: ungefähr die Hälfte der Kinder sieht diesen mindestens einmal wöchentlich; die Angaben zum Anteil jener, die diesen selten oder nie sehen, schwankt in den einzelnen Studien zwischen $10 \%$ und $50 \%$. Eine Obsorge beider Elternteile dürfte die

bei unverändertem Scheidungsverhalten durch eine Scheidung enden werden, d.h. 43 \% der im Jahr 2011 geschlossenen Ehen werden voraussichtlich geschieden. Dies bedeutet nicht - wie oft fälschlich interpretiert -, dass nahezu die Hälfte aller bestehenden Ehen geschieden werden.

${ }^{8}$ Berechnet auf Basis der Familien- und Haushaltsstatistik 2010, 56. Die Daten zu geschiedenen Personen in Lebensgemeinschaften wurden dankenswerterweise von Mag. Karin Klapfer (Statistik Austria) zur Verfügung gestellt.

${ }^{9}$ Laut Mikrozensus 2001 (KYTIR, SCHRITTWIESER 2003, 23) sah mehr als jedes fünfte Kind, das getrennt von seinem Vater lebte, diesen seltener als einmal im Jahr oder nie. $16 \%$ hatten täglich, $28 \%$ wöchentlich, $24 \%$ monatlich und $8 \%$ jährlich Kontakt. In der Studie von FIGDOR u.a. 2006, 197 hatten im ersten Jahr nach der Scheidung $4 \%$ selten und $5 \%$ gar keinen Kontakt. Ein Drittel ( $31 \%$ ) hatte täglich bis mehrmals pro Woche Kontakt, $21 \%$ mehrmals pro Woche und 2 Wochen- 
Kontaktfrequenz erhöhen (ATTENEDER u.a. 2005; FIGDOR u.a. 2006) und die Kontaktabbruchrate senken (BARTH-RICHARZ 2010).

Die häufigste Lebensform nach einer Scheidung bzw Trennung sind Ein-Eltern-Familien. Im Jahr 2011 stellten diese 12,3\% aller österreichischen Familien (10,5\% Mutter-Kind-Familien, 1,8 \% Vater-Kind-Familien). Jede achte Frau mit Kindern unter 15 Jahren ist alleinerziehend (13,1\%), ebenso $1 \%$ der Männer. Sowohl die Anzahl als auch der Anteil an Alleinerziehenden mit Kindern in dieser Altersgruppe ist seit 1985 nur leicht angestiegen und betrug zwischen 12 und $14 \%$ (alleinerziehende Mütter) bzw. ein bis knapp zwei Prozent (alleinerziehende Väter) (Statistik Austria 2012c, 25). 12 \% aller Kinder unter 15 Jahren leben in einer Ein-ElternFamilie, davon $94 \%$ bei der Mutter und $6 \%$ beim Vater (Statistik Austria 2012c, 70). Mütterliche Ein-Eltern-Familien sind mit einer Fülle an Herausforderungen konfrontiert, darunter eine stark erhöhte Armutsgefährdung (ZARTLER, BEHAM 2011).

Eine weitere häufige Lebensform nach einer Scheidung bzw Trennung sind Stieffamilien („Patchworkfamilien“), in welche Elternteile ihre Kinder aus früheren Beziehungen einbringen. Jede zehnte österreichische Familie mit Kindern ist eine Stieffamilie (Statistik Austria 2012c, 26). Die Hälfe (49\%) sind komplexe Stieffamilien (zumindest ein Partner hat ein Kind aus einer früheren Beziehung und ein gemeinsames Kind), und knapp jede zweite ist eine Steifvaterfamilie (44\%). Relativ selten sind

enden im Monat, $26 \%$ alle 14 Tage, $13 \%$ einmal im Monat bis mehrmals im Vierteljahr. In der Studie von TAZI-PREVE u.a. (2008) machten $40 \%$ der befragten Väter keine Angaben. Von den restlichen gab die Hälfte an, ihre Kinder mindestens einmal wöchentlich zu sehen, ein Viertel mindestens einmal im Monat und jeweils ein Zehntel selten bzw. nie. Die AutorInnen schätzen unter Einbezug der nicht Antwortenden, dass bis zu $50 \%$ der von ihnen befragten Väter keinen Kontakt haben.
Stiefmutterfamilien $(5 \%)$ sowie zusammengesetzte Stieffamilien (2\%; beide Partner bringen ein leibliches Kind mit, es gibt keine gemeinsamen Kinder). Jedes zehnte österreichische Kind unter 15 Jahren lebt in einer Stieffamilie. 889\% aller Stiefkinder leben bei der leiblichen Mutter, $11 \%$ beim Vater (Statistik Austria 2012c, 26f). Rund $43 \%$ aller Stieffamilien sind nichteheliche Lebensgemeinschaften. Während von allen Kindern mit verheirateten Eltern nur $7 \%$ in einer Stieffamilie leben, trifft dies auf ein knappes Drittel (30\%) der Kinder in nichtehelichen Lebensgemeinschaften zu (Statistik Austria 2012c, 80 und 85).

Einstellungsbefragungen zeigen eine steigende gesellschaftliche Akzeptanz von Scheidungen. So stimmen $83 \%$ der ÖsterreicherInnen der Aussage $\mathrm{zu}$, dass eine Scheidung im Allgemeinen die beste Möglichkeit zum Umgang mit unlösbaren Eheproblemen darstellt (WERNHART, NEUWIRTH 2007, 40). Auch die europäische Wertestudie zeigt, dass Scheidungen heute weniger sanktioniert werden als noch vor rund zwanzig Jahren: im Jahr 1990 fand ein Drittel (32\%) Scheidungen "keinesfalls in Ordnung“, 2008 ein Fünftel (22\%). Umgekehrt fand 1990 jede/r Fünfte $(19 \%)$ eine Scheidung "in Ordnung", 2008 waren es $30 \%$ (HAMACHERs-ZubA u.a. 2009). Für Familien mit (kleineren) Kindern sind die Einstellungen weniger permissiv, wie eine Auswertung des European Social Survey (2006/07) für Österreich zeigt:10 Die Scheidung eines Mannes mit Kindern unter zwölf Jahren wird von $39 \%$ der Befragten abgelehnt, jene einer Frau mit Kindern in diesem Alter von $29 \% .{ }^{11}$

Zusammenfassend betrachtet hat sich die Anzahl familialer Übergänge, die Eltern und Kin-

\footnotetext{
${ }^{10}$ Für die Durchführung der Auswertung danke ich Caroline Berghammer.

${ }^{11}$ Der geschlechtsspezifische Unterschied könnte auf traditionelle Vorstellungen über die Rolle des Vaters als Familienernährer hinweisen.
} 
der heute erleben, erhöht, und das Familienleben verläuft zunehmend als dynamischer Prozess. Der Ablauf des klassischen Familienzyklus (Eheschließung - Geburt von Kindern - Zusammenleben als Paar nach dem Auszug von Kindern bis zum Tod eines Partners) wird vielfach durchbrochen, und die biographischen Zeitanteile, die Menschen in einer Kernfamilie verbringen, verringern sich. Das Beziehungsgeflecht zwischen Eltern und Kindern wird mitunter aufgrund wiederholter Trennungen und neuer Partnerbeziehungen komplex, was die Gestaltung familialer Beziehungen $\mathrm{zu}$ einer Herausforderung machen kann.

\section{Rechtliche Regelung familien- dynamischer Prozesse nach Trennung und Scheidung: Diskrepanzen und Herausforderungen}

Aus den dargestellten Rahmenbedingungen resultieren Herausforderungen für das Rechtssystem, ${ }^{12}$ da sich mitunter Diskrepanzen zwischen normativen Richtlinien und der empirisch darstellbaren Alltagsrealität von Familien ergeben. Einige Bereiche werden im Folgenden beispielhaft angeführt.

\section{Rollenteilung: Partnerschaftsprinzip versus Ernährermodell}

Im österreichischen Rechtssystem ist die Gleichberechtigung von Mann und Frau in der Ehe und im Verhältnis zu ihren Kindern weitreichend verwirklicht. Auch auf der Einstellungsebene sind partnerschaftliche Prinzipien verankert: Die Akzeptanz einer strikt traditionellen Rollenteilung zwischen Mann und Frau hat stark abgenommen, partnerschaftliche Rollentei-

\footnotetext{
12 Siehe auch (FISCHER-CZERMAK u.a. 2008; HOPF 2011).
}

lung wird als gut und richtig wahrgenommen, und weibliche Berufsorientierung erfährt erhöhte Wertschätzung ${ }^{13}$ (PICKER u.a. 2005; SCHULZ, HUMMER 2005; WERNHART, NEUWIRTH 2007; HAMACHERS-ZUBA u.a. 2009).

Auf der Handlungsebene hingegen ist das partnerschaftliche Familienmodell nur bedingt vorfindbar: Österreich gehört zu jenen Ländern, in denen traditionelle Rollenteilungen zwischen Vätern und Müttern nach wie vor stark verbreitet sind. Das traditionelle männliche Ernährermodell („Hausfrauenehe“: Mann arbeitet Vollzeit, Frau ist nicht erwerbstätig) entwickelt sich nur langsam hin zu einem teilmodernisierten Ernährermodell (Mann Vollzeit, Frau Teilzeit) (HAAS 2009). In zwei Drittel aller Familien mit Kindern unter 15 Jahren wird eine Form des männlichen Ernährermodells gelebt. ${ }^{14}$ Trotz Veränderungen im väterlichen Rollenverständnis bleibt die materielle Versorgungsfunktion ein wesentlicher Bestandteil des Vaterbildes (zusammenfassend BEHAM, ZARTLER 2010). Der Übergang zur Elternschaft ist vielfach verbunden mit einer Retraditionalisierung der Rollenteilung und einer Erhöhung der Arbeitszeiten von Vätern (BEHAM, HALLER 2005; HOFINGER, ENZENHOFER 2006). Dies verweist auch auf Zwänge der Arbeitswelt und Arbeitskultur sowie auf unzureichende Rahmenbedingungen. Im Fall einer Scheidung spiegeln die zu treffenden obsorgerechtlichen Regelungen häufig die während aufrechter Ehe bestehende Rollenteilung wider. Auch bei Obsorge beider Elternteile bleibt in rund $90 \%$ der Fälle die Mutter der Residenz-Elternteil, insbesondere wenn die Kinder

\footnotetext{
${ }^{13}$ Dennoch wird die Berufstätigkeit von Müttern mit Kleinkindern sowie Kindern im Vor- und Pflichtschulalter nach wie vor äußerst skeptisch betrachtet (siehe im Überblick BEHAM, ZARTLER 2010).

$1443 \%$ leben das teilmodernisierte Ernährermodell, 23 \% das männliche Ernährermodell (Statistik Austria 2012c, 32).
} 
unter 14 Jahre alt sind (KreissL, PeliKan 2004; ATTENEDER u.a. 2005; FIGDOR u.a. 2006).

Geschlechtsspezifische Aspekte der Obsorgeregelungen wurden bereits bei Ermöglichung der Obsorge beider Elternteile mit Einführung des Kindschaftsrechts-Änderungsgesetzes $2001^{15}$ heftig diskutiert (LINDNER 2007), und auch der derzeit vorliegende Entwurf zum KindRÄG 2012 führt u.a. aufgrund der geplanten Ausweitungen der Obsorge beider Elternteile zu Diskussionen. Häufig wird vermutet, Obsorgefragen würden als Machtinstrument im Scheidungskonflikt missbraucht werden. ${ }^{16}$ Umfassende Studien über die Prozesse, welche zur Einigung auf ein Obsorgemodell17 führen, liegen allerdings ebenso wenig vor wie spezifische Einstellungsdaten zu Fragen der elterlichen Obsorge. Erste Auswertungen des Gender and Generations Survey 2008/0918 zeigen: die gesellschaftliche Meinung spiegelt die Polarisierung der Diskussion wider, mit einer Neigung zur mütterlichen Obsorge. Die Befragten (18 bis 45 Jahre alt) wurden gebeten, $z u$ folgender Aussage Stellung zu nehmen: „Wenn sich Eltern scheiden lassen, ist es besser, wenn das Kind bei der Mutter bleibt und nicht beim Vater." Jede/r Vierte (26\%) der Befragten stimmt (sehr) zu; ein Fünftel $(19,6 \%)$ stimmt (überhaupt) nicht $z u$, und mehr als die Hälfte der Befragten zeigt sich weder zustimmend noch ablehnend.

\footnotetext{
${ }^{15}$ BGBl I Nr. 135/2000, in Kraft seit 1. 7. 2001.

${ }^{16}$ In der Evaluierungsstudie von (FIGDOR u.a. 2006) wurde die Obsorgeregelung allerdings von den Befragten überwiegend als konsensual erlebt; lediglich 5 bis $8 \%$ der befragten Eltern fühlten sich vom anderen Elternteil unter Druck gesetzt.

${ }^{17}$ Eine Obsorge beider Elternteile setzt voraus, dass (1) dies dem Kindeswohl nicht entgegensteht, (2) ein hauptsächlicher Wohnort des Kindes vereinbart wird, (3) Konsens zwischen beiden Elternteilen besteht.

${ }^{18}$ Für die Durchführung der Auswertung danke ich Caroline Berghammer.
}

\section{Ehezentrierung}

Eine steigende Anzahl von Menschen lebt in rechtlich nicht institutionalisierten Partnerbeziehungen, dennoch schaffen eheliche und nichteheliche Lebensgemeinschaften unterschiedliche rechtliche Realitäten. Während im Kindschaftsrecht die Ungleichbehandlung von ehelichen und unehelichen Kindern nahezu beseitigt ist, bestehen weiterhin direkte Differenzierungen im Ehe- und Partnerschaftsrecht, und es gibt kaum rechtliche Sicherheiten für unehelich zusammenlebende Partner (MOTTL 2010, FISCHER-CZERMAK, BECLIN 2012). Einige Beispiele für Ungleichbehandlungen, welche aus dieser Ehezentrierung resultieren, werden im Folgenden genannt.

Eheliche und uneheliche Kinder sind hinsichtlich des Unterhaltsanspruchs gleichgestellt. Für den Unterhalt des betreuenden Elternteils wird hingegen strikt unterschieden, ob die Eltern miteinander verheiratet waren oder nicht: Der geschiedene Ehegatte hat (unabhängig von der Art der Scheidung und vom Verschulden), wenn es ihm aufgrund der Pflege und Erziehung eines gemeinsamen Kindes nicht zugemutet werden kann, sich selbst zu erhalten, Anspruch auf Betreuungsunterhalt bis zum fünften Lebensjahr des Kindes (§68a Abs. 1 EheG). Nicht verheiratete Väter haben hingegen gar keinen, nicht verheiratete Mütter lediglich einen Anspruch auf Unterhalt während der ersten sechs Wochen nach der Geburt ( $\$ 168$ ABGB). Das führt faktisch zu einer indirekten Ungleichbehandlung der Kinder: Nichteheliche Kinder müssen bereits im Säuglingsalter auf umfassende Betreuung durch einen Elternteil verzichten, wenn dieser aus ökonomischen Gründen aufgrund der Befristung des Betreuungsunterhalts gezwungen ist, einer Erwerbstätigkeit nachzugehen (NOWACK, GANNER 2010).

Die Obsorgeregelungen für verheiratete und unverheiratete Väter nach einer Trennung bzw Scheidung sind unterschiedlich. Die Eltern eines ehelichen Kindes haben von Beginn an ein Recht 
auf gemeinsame Obsorge, welche auch nach einer Trennung oder Scheidung grundsätzlich aufrecht bleibt. Widerspricht dieser Zustand dem Kindeswohl oder besteht kein Konsens zwischen den Eltern, so wird die alleinige Obsorge nach richterlicher Prüfung entweder der Mutter oder dem Vater übertragen. Die Obsorge für ein nichteheliches Kind steht hingegen nach $\S 166$ ABGB - außer im Fall der Kindeswohlgefährdung - automatisch der Kindesmutter zu. Um auch den Vater in die Obsorge einzubeziehen, bedarf es eines Antrags beider Elternteile und einer richterlichen Entscheidung. Mangels Zustimmung der Mutter besteht keine Möglichkeit, im Einzelfall zu prüfen, ob eine Obsorge beider Elternteile im Interesse des unehelichen Kindes wäre bzw. welcher Elternteil besser zur Ausübung einer alleinigen Obsorge geeignet wäre. Auf diese unterschiedlichen gerichtlichen Prüfungsmöglichkeiten, welche ehelichen und nichtehelichen Vätern eingeräumt werden, haben zuletzt zwei aufsehenerregende Urteile des Europäischen Gerichtshofs für Menschenrechte hingewiesen (Zaunegger gegen Deutschland, Sporer gegen Österreich) (VERSCHRAEGEN 2010; KOPETZKI, VAŠEK 2011). Die Behandlung ehelicher und unehelicher Eltern-Kind-Verhältnisse wird somit künftig Gegenstand legislativer Überlegungen sein.

Auch in der rechtlichen Regelung der Lebenssituation von Stieffamilien im Rahmen des Familienrechts-Änderungsgesetzes $2009^{19}$ wird eine Ehezentrierung deutlich. Das Gesetz unterscheidet zwischen ehelichen und nichtehelichen Stiefelternteilen: Während jenem Stiefelternteil, der mit dem leiblichen Elternteil verheiratet ist, eine Beistands- und Vertretungspflicht ${ }^{20}$ in Ob-

\footnotetext{
${ }^{19}$ BGBl. I 2009/75; in Kraft seit 1. 10. 2010

${ }^{20}$ Die Beistandspflicht umfasst die Pflege und Erziehung sowie Beaufsichtigung (z.B. Begleitung zum Arzt, Pflege bei Krankheit). Die Vertretungsbefugnisse regeln die Vertretung des leiblichen Elternteils
}

sorgeangelegenheiten des täglichen Lebens zukommt $^{21}$ (§90 Abs. 3 ABGB), hat der nicht verheiratete Stiefelternteil lediglich eine Schutzpflicht gegenüber dem Kind und damit „alles den Umständen nach Zumutbare zu tun, um das Kindeswohl zu schützen“ (§ 137 Abs. 4 ABGB); eine Handlungspflicht besteht nur, wenn das Kindeswohl gefährdet ist. Die Rechte und Pflichten nichtehelicher Stiefelternteile sind also deutlich weniger umfassend als jene verheirateter Stiefelternteile, denen u.U. sogar mehr Rechte zukommen als dem leiblichen, nicht obsorgeberechtigten Elternteil (BARTH-RICHARZ 2007). Diese Ungleichheiten stehen in krassem Widerspruch dazu, dass in fast der Hälfte aller Stieffamilien die Partner nicht verheiratet sind.

\section{Haushaltszentrierung}

Die österreichische Rechtslage weist in einigen Bereichen eine Haushaltszentrierung auf. So sind die obsorgerechtlichen Regelungen nach einer Scheidung damit verknüpft, einen hauptsächlichen Aufenthaltsort des Kindes festzulegen, an dem es sich mehr als $50 \%$ der Zeit aufhält (Residenzmodell). Verbringt das Kind bei beiden Elternteilen große Zeiträume, so können die rechtlichen Rahmenbedingungen nicht entsprechend angepasst werden (etwa unterhaltsrechtliche Folgen, Auszahlung der Familienbeihilfe). Für Wechselmodelle (Pendelmodell, Doppelresidenz), in welchen die Kinder tageoder wochenweise zwischen beiden Elternteilen wechseln, existiert bislang keine gesetzliche Grundlage - auch wenn diese von einem kleinen Teil geschiedener Eltern gelebt werden (BARTHRICHTARZ 2009b; BARTH-RICHTARZ 2009a).

Ein weiterer Bereich, in dem aufgrund der Haushaltszentrierung familiendynamische Pro-

gegenüber Dritten (z.B. Unterschrift einer Entschuldigung für die Schule).

${ }^{21}$ Dies gilt nur, wenn der leibliche Elternteil die Obsorge für dieses Kind hat (alleinige Obsorge oder Obsorge beider Elternteile). 
zesse nicht entsprechend berücksichtigt werden, ist die elterliche Pflegefreistellung im Krankheitsfall. Derzeit ist es nur dem ResidenzElternteil möglich, eine Pflegefreistellung in Anspruch zu nehmen, was eine massive Benachteiligung von Alleinerziehenden darstellt: selbst wenn der andere Elternteil bereit wäre, eine Pflegefreistellung in Anspruch zu nehmen, gibt es dafür keine rechtliche Grundlage. Eine solche Möglichkeit würde die Kinderbetreuung im Krankheitsfall für Alleinerziehende besser organisierbar machen.

\section{Position des Kindes nach einer elterlichen Scheidung/Trennung}

Das Kindeswohl gilt als Leitprinzip im Kindschaftsrecht, und generell ist eine Stärkung der Stellung des Kindes im Zuge einer elterlichen Scheidung festzustellen. Maßnahmen wie die Einführung des Kinderbeistands sowie die persönliche Anhörung von Minderjährigen in Verfahren über Pflege und Erziehung oder das Recht auf persönlichen Verkehr tragen dazu bei (ZARTLER, WILK 2010, sowie iFamZ 4/2010). Dennoch ist teilweise eine relativ geringe Fokussierung auf die Wahrnehmung kindlicher Interessen festzustellen.

So wäre beispielsweise $\mathrm{zu}$ fragen, ob die Berechnung des Kindesunterhalts den aktuellen Bedürfnissen von Kindern entspricht und welche Aussagekraft die Regebedarfssätze ${ }^{22}$ für die tatsächlichen Kinderkosten besitzen. Die Festsetzung der Regelbedarfssätze beruht auf einer Kinderkostenanalyse auf Basis der Konsumerhebung 1964 (Statistische Nachrichten 1970/4), wobei lediglich eine Indexanpassung erfolgt, Verschiebungen von Ausgabenstruktur und Konsumverhalten aber nicht berücksichtigt werden (BUCHEGGER, WÜGER 2004; WEITZENBÖCK

\footnotetext{
${ }^{22}$ Die Regelbedarfssätze bilden, unabhängig von den konkreten Lebensverhältnissen der Eltern, einen allgemeinen Richtsatz für den mittleren Bedarf von Kindern einer bestimmten Altersstufe.
}

2004; PÖHLMANN 2005; GITSCHTHALER 2008). Eine Evaluierung der Ermittlung und Höhe des Regelbedarfs, der Abgrenzung zum Sonderbedarf sowie der Sinnhaftigkeit der Obergrenze (2,5facher Regelbedarf) erscheint angebracht.

In der sozialwissenschaftlichen Forschung besteht Einigkeit über die positiven Auswirkungen der Aufrechterhaltung des Kontakts zu beiden Elternteilen auf die kindliche Entwicklung23 (SCHNEEWIND, WALPER 2008). Dennoch haben geschiedene Elternteile zwar ein Besuchsrecht, aber keine Verpflichtung, den Kontakt zu ihrem Kind aufrecht zu erhalten (JAUSOVEC 2009; BECK 2010). Die Erzwingung von Kontakten gegen den Willen des Besuchsberechtigten ist nicht möglich (§ 108 AußStrG). Argumentiert wird, dass eine Durchsetzung mit Zwangsmitteln nicht dem Kindeswohl dienen würde (Pesendorfer 2011). Während also geschiedenen Eltern ein Besuchsrecht zukommt, verfügen ihre Kinder über kein (durchsetzbares) Rechtsmittel, den getrennt lebenden Elternteil kennen zu lernen bzw mit ihm Kontakt zu halten, wenn dieser desinteressiert ist. Auch auf Elternebene besteht diesbezüglich ein Ungleichgewicht: Es ist sanktionslos, wenn ein Elternteil sein Besuchsrecht nicht wahrnimmt. Umgekehrt gibt es aber Zwangsmittel (Geldstrafen, Beugehaft) für jenen Elternteil, der das Besuchsrecht vereitelt (PESENDORFER 2011, 66). ${ }^{24}$

\footnotetext{
${ }^{23}$ Mit wenigen Ausnahmen wie Gewalt, extrem konflikthafte Beziehung der Eltern, schwere psychische Erkrankung oder Suchterkrankung (FIGDOR 2011). ${ }^{24}$ In einem OGH-Urteil von April 2011 (4 Ob 8/11x) wurde sogar einem Vater aufgrund eines Verstoßes der Mutter gegen das Wohlverhaltensgebot Schadenersatz zugesprochen. Schäden, die Kinder aufgrund der Verletzung von Verhaltenspflichten der Eltern erleiden, sind bislang nicht einklagbar.
} 


\section{Berücksichtigung familiendynamischer Prozesse}

Familien sind nicht statisch, sondern entwickeln und verändern sich im Zeitverlauf, u.a. aufgrund von Trennungen bzw Scheidungen, dem Eingehen neuer Partnerschaften und dem Entstehen multipler Eltern-Kind-Verhältnisse. Solche familiendynamischen Prozesse finden im Recht teilweise zu wenig Berücksichtigung. Beispielhaft seien die Regelungen zum Bezug des Kinderbetreuungsgeldes genannt. Nach der Geburt eines Kindes können die Eltern gemäß dem mit 1. Jänner 2010 in Kraft getretenen Kinderbetreuungsgeldgesetz zwischen fünf unterschiedlichen Bezugsmodellen wählen; die Höhe des Kinderbetreuungsgeldes hängt von der Bezugsdauer ab. Das bei der erstmaligen Antragstellung gewählte Modell ist für beide Elternteile bindend und kann in der Folge nicht mehr geändert werden. Wählt nun ein Paar - im Vertrauen auf das kontinuierliche Einkommen des Partners und die Haltbarkeit der Beziehung ein längeres Bezugsmodell mit geringerer monatlicher Bezugshöhe, so kann dies auch dann nicht revidiert werden, wenn sich die Rahmenbedingungen beispielsweise durch eine Trennung verändern. Die finanziellen Mittel für den/ die AlleinerzieherIn, welche/r das Kind hauptsächlich betreut, sind dann ausgesprochen gering (rund 436 Euro monatlich in der längsten Bezugsvariante ${ }^{25}$. Diese Inflexibilität trifft unverheiratete Eltern umso härter, als nach einer Trennung kein Anspruch auf Betreuungsunterhalt besteht (s.o.).

${ }^{25}$ Auch hinsichtlich der Bezugsdauer des Kinderbetreuungsgeldes zeigt sich eine Diskriminierung von Alleinerziehenden, da für sie die Wahl eines längeren Modells mit Beteiligung des Partners nicht möglich ist - selbst wenn im Verlauf der Bezugsdauer die Partnerbeziehung wieder aufgenommen wird. Daraus resultiert eine Benachteiligung der Kinder von getrennt lebenden Eltern, denen eine kürzere Betreuungsdauer zusteht als jenen, deren Eltern $\mathrm{zu}^{-}$ sammenleben.
Weiterer Handlungsbedarf zeichnet sich im Zusammenhang zwischen Scheidung und Mobilität ab. Aufgrund veränderter gesellschaftlicher Rahmenbedingungen (Ansteigen beruflicher Mobilitätsanforderungen, Verbreitung neuer Kommunikationstechnologien) sind Partnerschaftsformen, welche Mobilität einschließen, im Steigen begriffen. So leben in Deutschland bereits $16 \%$ der erwerbsfähigen Bevölkerung aus beruflichen Gründen in einer mobilen Lebensform (LAT) (SCHNEIDER u.a. 2002; LIMMER 2005). In Österreich lebt ein Fünftel der 18- bis 45Jährigen in LAT-Partnerschaften (BAIERL 2009), und in rund einem Viertel aller neu geschlossenen Ehen sind ausländische StaatsbürgerInnen beteiligt. ${ }^{26}$ Im Trennungs- bzw. Scheidungsfall ergeben sich hier komplexe Herausforderungen für die Organisation der Eltern-Kind-Kontakte sowie in Bezug auf das Aufenthaltsbestimmungsrecht. In internationalen Abkommen ${ }^{27}$ ist geregelt, dass ein Kind nicht ohne Zustimmung des anderen obsorgeberechtigten Elternteils in ein anderes Land gebracht werden darf. Diese gesetzlichen Grundlagen wurden mit der Intention entwickelt, das Verbringen („Entführen“) eines Kindes in das Heimatland des besuchsberechtigen Elternteils (meist: des Vaters) zu verhindern. Mittlerweile zeigt sich allerdings: die überwiegende Zahl der Fälle betrifft obsorgeberechtigte Mütter, die ihre Kinder verbringen, weil sie in ihren Heimatstaat zurück-

\footnotetext{
${ }^{26}$ In $18 \%$ der im Jahr 2010 geschlossenen Ehen war einer der beiden Partner nicht österreichische/r StaatsbürgerIn, in $5 \%$ galt dies für beide Partner. Drei Viertel $(77 \%)$ der Ehen wurden zwischen InländerInnen geschlossen.

(http://www.statistik.at/web_de/statistiken/bevoelker ung/eheschliessungen/023945.html), eingesehen am 25. 8. 2011. Im Jahr 1970 fanden $94 \%$ aller Eheschließungen zwischen ÖsterreicherInnen statt, $5 \%$ waren binational, und in 0,6 \% der Fälle hatten beide Partner nicht die österreichische Staatsbürgerschaft (Statistik Austria 2008, 143).

${ }^{27}$ Haager Kindesentführungsübereinkommen, Brüssel-IIa-Verordnung.
} 
kehren wollen (MiKLAU 2010). Komplexe Abwägungen und Verflechtungen zwischen dem Wohl des Kindes, seiner (Halb- bzw. Stief-) Geschwister sowie beider Elternteile sind die Folge (MÖCKLI 2011; Traar 2011).

\section{Abschließende Bemerkungen}

Rechtliche Leitbilder und Definitionen von Familie beeinflussen ganz massiv die alltägliche Lebensrealität von Familien. Sie legen fest, welche Lebensform als Familie anerkannt und zivilrechtlich, sozial(versicherungs-)rechtlich oder steuerrechtlich berücksichtigt, begünstigt oder belastet wird. Zwischen den normativen Vorgaben des Familienrechts und empirischen Befunden der Familienforschung besteht ein enges Wechselverhältnis, das nicht ganz spannungsfrei ist, wie exemplarisch gezeigt wurde. Die rechtlichen Konzeptionen stimmen vielfach nur bedingt mit der demographisch und soziologisch darstellbaren Situation überein, wobei die Zusammenhänge in unterschiedliche Richtungen gehen. Während das Recht in einigen Bereichen an empirisch überholten Konzepten festhält (beispielsweise Ehe- und Haushaltszentriertheit), schafft es in anderen Bereichen rechtliche Normen, die der familialen Alltagsrealität voraus sind (beispielsweise partnerschaftliche eheliche Rollenteilung).

Eine angemessene rechtliche Normierung des Familienlebens erfordert aufgrund des raschen familialen Wandels einen differenzierten $\mathrm{Zu}$ gang. Auch wenn in Zukunft keine gänzlich neuen Lebensformen hinsichtlich formaler struktureller Merkmale erwartet werden können, wird die individuelle Binnengestaltung von Lebensformen vielfältiger werden (Burkart 2009). Die Lebensverläufe von Menschen werden zunehmend davon gekennzeichnet sein, „dass sich stabile Phasen im Lebenslauf abwechseln mit unsicheren Lebensformen, die teils freiwillig, aber auch ungeplant entstehen. Hier ist das Rechtssystem gefordert, sich stärker als bisher auf diese diskontinuierliche Dynamik in den Lebensläufen einzustellen. Der Lebenslauf folgt immer seltener einem vorhersehbaren Verlauf und wird damit immer schwerer durch generelle Regelungen fassbar." (SCHNEIDER, MATTHIAS-BLECK 1999, 207)

Rechtliche Bemühungen, welche der aktuellen Lebensrealität von Familien entsprechen, sind daher künftig gefordert, Unsicherheiten, Instabilitäten und diskontinuierliche Dynamiken ebenso $\mathrm{zu}$ berücksichtigen wie die zunehmend geringere Ausrichtung von Familien an der Ehe oder am gemeinsamen Haushalt. Ein zentraler Bereich müsste die Berücksichtigung der Perspektive von Kindern sowie die Schutz- und Förderungswürdigkeit der Eltern-Kind-Beziehung sein, wobei Maßnahmen, die dem Kindeswohl dienen, auch in der Komplexität ihrer Auswirkungen auf das gesamte Familiensystem zu sehen wären. 


\section{Abkürzungen:}

$\begin{array}{ll}\text { AußStrG } & \begin{array}{l}\text { Außerstreitgesetz } \\ \text { BMJ }\end{array} \\ \text { BMWJF } & \begin{array}{l}\text { Bundesministerium für Justiz } \\ \text { Jugend und Familie }\end{array} \\ \text { EPG } & \begin{array}{l}\text { Eingetragene Partnerschaftsgesetz } \\ \text { interdisziplinäre Zeitschrift für }\end{array} \\ \text { iFamZ } & \begin{array}{l}\text { Familienrecht } \\ \text { Kindschaftsrechtsänderungsgesetz }\end{array} \\ \text { KindRÄG } & \text { Living Apart Together } \\ \text { LAT } & \text { Nichteheliche Lebensgemeinschaft } \\ \text { NEL } & \end{array}$

Siehe auch das allgemeine Abkürzungsverzeichnis: http://www.rechtsgeschichte.at/beitraege/abk.pdf

\section{Literatur:}

Christine AtTENEDER u.a. (2005), Auswirkungen von Scheidung auf Kinder, Frauen und Männer (vor dem Hintergrund des Eherechts-Änderungsgesetzes 1999 und des KindschaftsrechtsÄnderungsgesetzes 2001) (Linz).

Andreas BAIERL (2009), LAT-Partnerschaften, in: Vienna Institute of Demography \& Österreichisches Institut für Familienforschung (Hg.), Familienentwicklung in Österreich. Erste Ergebnisse des "Generations and Gender Survey (GGS)" 2008/09 (Wien) 25.

Judith BARTH-RICHARZ (2007), Obsorge der Stiefeltern für ihre Stiefkinder. Meinungen von Experten/ Expertinnen, in: iFamZ 2/3, 115-118.

Judith BARTH-RICHARZ (2010), Neue empirische Ergebnisse zur gemeinsamen Obsorge, in: Interdisziplinäre Zeitschrift für Familienrecht 5/3, 126-128.

Judith BARTH-RICHARZ (2009a), Die Doppelresidenz nach Trennung und Scheidung. Ein ideales Modell? - Meinungen von Experten, in: iFamZ 4/3, 178-181.

Judith BARTH-RICHARZ (2009b), Zwei "Zuhause" für unsere Kinder. Erfahrungen von Müttern und Vätern mit der Doppelresidenz nach Trennung/ Scheidung, in: iFamZ 4/3, 174-177.

Susanne BECK (2010), BesuchsRecht - DurchsetzungsPflicht, Vortragsmanuskript, Familienrichtertag 2010.

Elisabeth BECK-GERNSHEIM (2009), Das ganz normale Chaos der Familie und des Familienrechts, in: Bundesministerium für Justiz (Hg.), Lebensform Familie. Realität und Rechtsordnung (Wien) 2332.
Martina BEHAM (2010), Familiengründung heute, in: Bundesministerium für Wirtschaft, Familie und Jugend (Hg.), 5. Österreichischer Familienbericht 1999-2009. Die Familie an der Wende zum 21. Jahrhundert, Bd. 1 (Wien) 225-258.

Martina BeHAM, Roland HALLeR (2005), Work-LifeBalance - Wie bringen Österreichs Familien Beruf und Familie in Einklang? in: Wolfgang SCHULZ, Max Haller, Alfred Grausgruber (Hgg.), Österreich zur Jahrhundertwende (Wiesbaden) 401-433.

Martina BEHAM, Ulrike ZARTLER (2010), Eltern und Kinder. Ansprüche, Anforderungen und Ambivalenzen in betreuungsintensiven Lebensphasen, in: Bundesministerium für Wirtschaft, Familie und Jugend (Hg.), 5. Österreichischer Familienbericht 1999-2009. Die Familie an der Wende zum 21. Jahrhundert, Bd. 1 (Wien) 363-402.

Geneviève BOUCHARD (2006), Cohabitation versus Marriage: The Role of Dyadic Adjustment in Relationship Dissolution, in: Journal of Divorce and Remarriage, 46/1-2, 107-118.

Reiner BUCHEGGER, Michael WÜGER (2004), Private Ausgaben für Kinder, in: Österreichischer Amtsvormun 36/180, 284-293.

Günter BURKART (2009) (Hg.), Zukunft der Familie. Prognosen und Szenarien (Opladen-Farmington Hills).

Helmuth FIGDOR (2011), Welche Gründe sprechen gegen die Obsorge beider Eltern? Kriterien für Entscheidungen über Obsorge und persönliche Kontakte des Kindes, in: iFamZ 6/3, 131-138.

Helmuth FIGDOR u.a. (2006), Evaluationsstudie über die Auswirkungen der Neuregelungen des KindRÄG 2001, insbesondere der Obsorge beider Eltern (Wien).

Constanze Fischer-CZERMAK, u.a. (2008) (Hgg.), ABGB 2011. Chancen und Möglichkeiten einer Zivilrechtsreform (Wien).

Constanze FISCHER-CZERMAK, Barbara BECLIN (2012) (Hgg.), Neue Regelungen für nichteheliche Lebensgemeinschaften? Verhandlungen des Achtzehnten Österreichischen Juristentags (Wien).

Andreas GESTRICH (2008), Sozialgeschichte der Familie in der Neuzeit, in: Norbert F. SCHNEIDER, Lehrbuch Moderne Familiensoziologie (OpladenFarmington Hills) 79-98.

Edwin GITSCHTHALER (2008), Unterhaltsrecht (Wien).

Barbara HAAS (2009), Geschlechtergerechte Arbeitsteilung - theoretisch ja, praktisch nein! Eine Bilanz für österreichische Paarhaushalte mit Kindern, in: Erna APPELT (Hg.), Gleichstellungspolitik in 
Österreich. Eine kritische Bilanz (InnsbruckWien-Bozen).

Ursula HAMACHERS-ZUBA, Erich LEHNER, Claudia TSCHIPAN (2009), Partnerschaft, Familie und Geschlechterverhältnisse in Österreich, in: Christian FrIESL, Ursula HAMACHERS-ZuBA, Regina POLAK (Hgg.), Die ÖsterreicherInnen. Wertewandel 1990-2008 (Wien) 87-142.

Paul B. HILL, Johannes KOPP (2006), Familiensoziologie. Grundlagen und theoretische Perspektiven (Wiesbaden).

Christoph HOFINGER, Edith ENZENHOFER (2006), Mehr Beruf, weniger Familie? Zur Lage der berufstätigen Väter in Österreich, in: Harald Werneck, Martina BehaM, Doris Palz (Hgg.), Aktive Vaterschaft. Männer zwischen Familie und Beruf (Gießen) 68-79.

Gerhard Hopf (2011), Reformen und Reformbedarf des österreichischen Familienrechts vor dem Hintergrund europäischer Entwicklungen, in: Tomislav BORIĆ u.a. (Hgg.), Öffnung und Wandel. Die internationale Dimension des Rechts II. Festschrift für Willibald Posch zum 65. Geburtstag. Wien: Lexis Nexis, 241-256.

Johannes HuININK, Dirk KONIETZKA (2007), Familiensoziologie. Eine Einführung (FrankfurtNew York).

Sybille JAUSOVEC (2009), Das Besuchsrecht zwischen Eltern und Kindern (Wien).

Christian KOPETZKI, Markus Vašek (2011), Ausschluss einer gerichtlichen Einzelfallprüfung der Obsorgeregelung diskriminiert den Vater eines unehelichen Kindes, in: iFamZ 6/2, 61-63.

Reinhard KReIsSL, Christa PeliKan (2004), Schutz vor Übervorteilung in Scheidungsverfahren (Wien).

Josef KYTIR, Karin SCHRITTWIESER (2003), Familienstrukturen und Familienbildung. Ergebnisse des Mikrozensus September 2001 (Wien).

Ruth LIMMER (2005), Berufsmobilität und Familie in Deutschland, in: Zeitschrift für Familienforschung, 17/2, 8-26.

Patrizia LINDNER (2007), Mein Kind, dein Kind, unser Kind. Eine wissenssoziologische Diskursanalyse rund um die Einführung der Obsorge beider Elternteile nach einer Scheidung (Dipl.arb. Univ. Wien).

Philipp MAYRING (2003), Qualitative Inhaltsanalyse. Grundlagen und Techniken (Weinheim-Basel).

Christine MikLaU (2010), Gemeinsame Obsorge, Kindesentführung und VO Brüssel IIa, in: Interdisziplinäre Zeitschrift für Familienrecht, 5/3, 133-140.
Michael MitTerauer (2009). Sozialgeschichte der Familie. Kulturvergleich und Entwicklungsperspektiven (Wien).

Urs Peter MöcKLI (2011). Zum Kindeswohl als Leitmaxime bei Rückführungsanordnungen, in: Interdisziplinäre Zeitschrift für Familienrecht, 6/3, 124-129.

Ingeborg MotTL, (2010). Familienrecht - Ausgangslage und Neuerungen, in: Bundesministerium für Wirtschaft, Familie und Jugend (Hgg.), 5. Österreichischer Familienbericht 1999-2009. Die Familie an der Wende zum 21. Jahrhundert, Bd. 2 (Wien) 5-90.

Rainer MÜNZ, Albert F. REITERER, (2010). Demografische Entwicklung im 20. Jahrhundert, in: Bundesministerium für Wirtschaft, Familie und Jugend (Hg.) 5. Österreichischer Familienbericht 1999-2009. Die Familie an der Wende zum 21. Jahrhundert, Bd. 1 (Wien) 1-90.

Nina NOWACK, Michael GANNER (2010). Verfassungswidrigkeit des Betreuungsunterhalts und des Unterhaltsanspruchs der nicht verheirateten Mutter? Ist eine Anpassung des Unterhaltsrechts erforderlich? in: iFamZ, 5/2, 68-71.

Ramona Faith OswALD, Katherine A. KuvAlanKA (2008), Same-Sex Counples. Legal Complexities, in: Journal of Family Issues, 29/8, 1051-1066.

Ulrich PESENDORFER (2011), Die Durchsetzung des Besuchsrechts. Rechtslage - allgemeine Folgen unterbliebener Kontakte - Rechtsvergleich Lösungsansätze, in: interdisziplinäre Zeitschrift für Familienrecht, 6/2, 64-71.

Ruth PicKer, Sigrid Nitsch, Eva Zeglovits (2005), Frauenstudie 2005: Österreicherinnen von 40 bis 60 Jahren (Wien).

Thomas PÖHLMANN (2005), Der Regelbedarf - eine (un)brauchbare Mogelpackung? in: Der österreichische Amtsvormund, 37/187, 223-233.

Klaus A. SCHNEEWIND, Sabine WALPER (2008), Kinder in verschiedenen Familienformen, in: Marcus HASSELHORN, Rainer K. Silbereisen (Hgg.), Entwicklungspsychologie des Säuglings- und Kindesalters (Göttingen) 571-616.

Norbert F SCHNEIDER, Ruth LIMMER, Kerstin RUCKDESCHEL (2002), Mobil, flexibel, gebunden. Familie und Beruf in der mobilen Gesellschaft (Frankfurt am Main).

Norbert SCHNEIDER, Heike MATTHIAS-BLECK (1999), Moderne Familie - altes Recht? Zur Situation des am überkommenen Normalitätskonzept von Familie und Lebenslauf orientierten Rechtssystems aus soziologischer Perspektive, in: Friedrich W. BUSCH, Bernhard NAUCK, Rosemarie 
NAVE-Herz (Hgg.), Aktuelle Forschungsfelder der Familienwissenschaft (Würzburg) 185-210.

Wolfgang Schulz, Christian HuMMer, (2005), Veränderungen in den Formen des Zusammenlebens und Wandel der Einstellungen zu Ehe und Familie, in: Wolfgang Schulz, Max HALler, Alfred GRAUSGRUBER (Hgg.), Österreich zur Jahrhundertwende. Gesellschaftliche Werthaltungen und Lebensqualität 1986-2004 (Wiesbaden) 343-366.

Reinhard SIEDER (1985), Sozialgeschichte der Familie. (Frankfurt am Main).

Statistik Austria (2008) (Hg.), Demographisches Jahrbuch 2007 (Wien).

Statistik Austria (2012a) (Hg.), Statistisches Jahrbuch 2012 (Wien).

Statistik Austria (2012b) (Hg.), Ehescheidungen. Pressemitteilung 10.268-121/12 (Wien).

Statistik Austria (2012c) (Hg.), Familien- und Haushaltsstatistik 2011 (Wien).

Miriam Irene TAZI-PREVE, Markus KAINDL, Benedikt KRENN, (2008), Väter ohne Kinder. Charakteristika von Scheidungsvätern ohne Kontakt zu ihren Kindern, in: Zeitschrift für Bevölkerungswissenschaft 33/1, 5-36.

Thomas TRAAR (2011), Bevorstehende Neuordnung des internationalen Kinderschutzes durch das KSÜ, in: iFamZ 6/2, 97-101.

Bea Verschraegen (2010), Elterliche (Ob-)Sorge Regel und Ausnahme: Wer bestimmt, wer entscheidet? in: iFamZ 5/1, 4-9.

Johann WEITZENBÖCK (2004), Die Kinderkostenanalyse und ihre (möglichen) Auswirkungen auf die Unterhaltsjudikatur, in: Österreichischer Amtsvormund 36/180, 293-299.

Georg WeRnHART, Norbert NeUWIRTH (2007), Geschlechterrollenwandel und Familienwerte (19882002), Österreich im europäischen Vergleich. Ergebnisse auf Basis des ISSP 1988, 2002. Working Paper (Wien).
Ulrike ZARTLER (2012), Die Kernfamilie als Ideal. Zur Konstruktion von Scheidung und Nachscheidungsfamilien, in: Zeitschrift für Familienforschung 24/1, 67-84.

Ulrike ZARTLER, Martina BeHAM (2011), Alleinerziehen. Alltägliche Herausforderungen im Umgang mit knappen Ressourcen, in: SWS-rundschau 51/4, 383-404.

Ulrike ZARTLER, Liselotte WILK (2010), Dynamiken und Veränderungen im Familienverlauf: Scheidung und Trennung, in: Bundesministerium für Wirtschaft, Familie und Jugend (Hg.), 5. Österreichischer Familienbericht 1999-2009. Die Familie an der Wende zum 21. Jahrhundert, Bd. 1 (Wien) 443-502.

\section{Interviews}

Peter BARTH, Familienrechtliche Abteilung des BMJ.

Susanne BECK, Familienrichterin.

Astrid DEIXLER-HÜBNER, Universität Linz.

Ewald FILLER, Ressort Familienrecht des BMWFJ, Kinder- und Jugendanwalt.

Michael HeNGL, Familienrichter.

Gerhard Hopf, Sektionschef des BMJ i.R.

Helene KLAAR, Rechtsanwältin.

Franz MAUTHNER, Familienrichter.

Klaus MEßNER, Familienrichter.

Christine MIKLAU, Richterin, Referentin am BMJ.

Michael STICH, Familienrichter.

Doris TÄUBEL-WEINREICH, Familienrichterin, Vorsitzende der Fachgruppe Familienrecht der österreichischen Richtervereinigung.

Gabriela THOMA-TWAROCH, Familienrichterin, Vorsteherin des BG Josefstadt.

Gabriele VANA, Rechtsanwältin. 